\title{
BOMBEO SOLAR DE ALTA POTENCIA CON PRESION Y CAUDAL CONSTANTE. CASO DE ÉXITO
}

\author{
Omaña Álvarez J.M. (1), Antonio de Castro. (2), Medrano Villalón J. (3)
}

(1) Ing. Agrónomo. Dpto. de Trasferencia tecnológica. AIMCRA (Asociación de Investigación para la mejora del cultivo de la remolacha azucarera). Crta. Villabañez, 201,47012 Valladolid. Correo: j.m.omana@aimcra.es

(2) Ing. Industrial e Ing. de Telecomunicaciones. Director de Riego Solar. C/Felipe Sánchez Román, $192^{\circ} \mathrm{C}, 47008$ Valladolid; Correo: info@riegosolar.net

(3) Agricultor. Lic. en Filosofía y Letras, Cooperativa Estrella de S. Juan. Villavieja del Cerro, Valladolid. Correo: justino.medra@gmail.com

\section{Resumen}

Fruto del impulso de AIMCRA y de la colaboración entre las empresas RiegoSolar y la cooperativa Estrella de S. Juan, se ha hecho realidad un proyecto de riego y energía de gran impacto innovador. La puesta en marcha de un demostrador de bombeo solar directo de alta potencia con presión y caudal constante, con el que se ha conseguido reducir el coste del riego en un $80 \%$ y las emisiones de $\mathrm{CO}_{2}$ a la atmosfera en un $100 \%$.

El sistema patentado por la empresa española de ingeniería RiegoSolar, es capaz de mantener la presión constante en riegos por aspersión de cualquier tamaño, tanto coberturas como pivotes o cañones, sin recurrir al uso de energías convencionales, ni al almacenamiento de energía en baterías o de agua en grandes balsas.

En este demostrador, basado en el uso exclusivo de la energía solar, se consigue también mejorar la eficiencia energética e hidráulica de la instalación, automatizar por completo la gestión del riego y realizar la programación de los riegos en base a las necesidades reales de los cultivos.

La instalación se localiza Torrecilla de la Abadesa (Valladolid), y ya ha funcionado a pleno rendimiento durante una campaña completa. Otras cuatro instalaciones más, de características similares, han entrado en funcionamiento durante el primer semestre de 2015.

\section{Introducción.}

La energía solar y el riego

Dentro de los usos posibles de la energía solar fotovoltaica, tal vez el riego sea uno de los que mejor se adaptan a este tipo de energía fluctuante. La energía generada en los paneles fotovoltaicos y las necesidades de agua de los cultivos, están regidas por la misma variable, la radiación solar; a mayor insolación mayores necesidades de agua del cultivo, pero también mayor capacidad para producir energía fotovoltaica. Cuanto más se necesita regar, más energía fotovoltaica está disponible para el bombeo.

En la Grafico $n^{0} 1$ se muestra la potencia fotovoltaica obtenida por radiación solar con una inclinación óptima durante los meses del año en España. Los meses de mayor radiación 
solar coinciden con los de mayores necesidades de agua para el cultivo, por lo que en bombeo directo podemos aprovechar casi un $70 \%$ de la radiación anual.

\section{POTENCIA DISPONIBLE Kwh/m²}

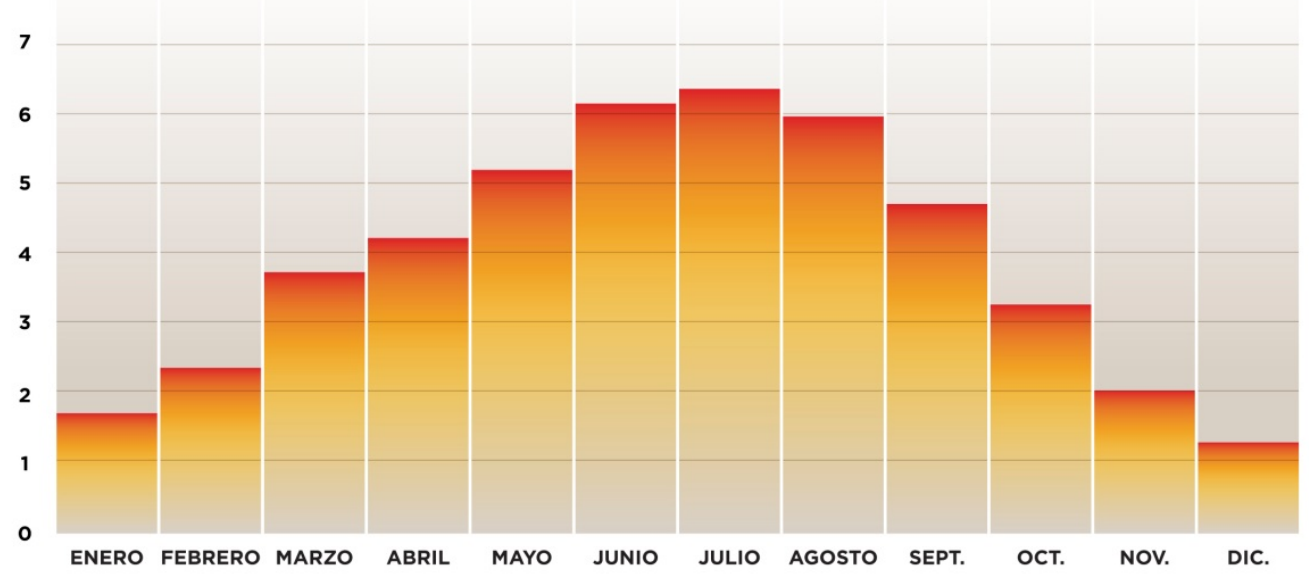

Grafico 1. Potencia disponible en $\mathrm{kwh} / \mathrm{m}^{2}$

Una oportunidad que debemos aprovechar

La agricultura es uno de los principales demandantes de energía de la sociedad, la extracción del agua desde captaciones subterráneas o superficiales, junto con la aplicación de la presión necesaria para el riego, supone un gran gasto de energía y un coste cada vez mayor.

La drástica bajada de precios de los paneles fotovoltaicos, junto con la tendencia al alza en el precio de los combustibles fósiles y la electricidad de red, han hecho que hayan ido apareciendo en el mercado sistemas de bombeo fotovoltaico que resultan rentables, aunque hasta ahora su aplicación se limitaba al abastecimiento para consumo humano o al riego por goteo o gravedad en pequeñas superficies.

En el caso del riego por aspersión aún quedaba un problema por resolver; conseguir mantener la presión y el caudal constante, es decir que no oscilasen con las variaciones de la radiación a lo largo del día.

Para poder mantener la presión constante hasta ahora no había más solución que almacenar el agua requerida por el cultivo para un día de riego, lo que se hacía principalmente de dos formas, mediante depósitos elevados donde la altura de la caída del agua aportaba la presión necesaria, o mediante balsas y una segunda bomba alimentada por fuentes de energía convencionales.

Esto presentaba el inconveniente de disponer de una elevada capacidad de almacenamiento de grandes cantidades de agua, lo que limitaba su aplicación a pequeñas superficies de cultivo, sin olvidarnos del consumo de energía, proporcionada por la red eléctrica o el gasóleo, de la segunda bomba encargada de aportar presión al agua embalsada. 


\section{Descripción del proyecto}

\section{Carácter innovador del proyecto de RiegoSolar}

RiegoSolar es el primer bombeo solar directo que proporciona presión y caudal constantes y que es compatible con cualquier tamaño de finca, profundidad de pozo o sistema de riego, ya que solo requiere adaptar la potencia de sus componentes a las necesidades.

El sistema está especialmente indicado para regar por aspersión grandes extensiones, ya sea mediante pivotes, coberturas o cañones, en los que se necesita mantener la presión constante a pesar de las variaciones de radiación solar que se producen a lo largo del día o de la época del año. Este punto es importante para poder llegar al grueso de la agricultura de muchas regiones en las que se riegan por aspersión cultivos extensivos, como la remolacha azucarera.

Otra de las aportaciones del sistema podríamos denominarla "riego inteligente". Mediante el cálculo de la ETc y la monitorización de los riegos y lluvias, se realiza el balance hídrico y la programación de los riegos, y mediante el empleo de automatismos se ejecutan los riegos con gran precisión y comodidad para el agricultor. La parcela se riega sola, con la frecuencia y la cantidad de agua que cada cultivo necesita en cada momento.

Finalmente se ha innovado en la eficiencia energética de la instalación, mediante el empleo de nuevos emisores en los pivotes se consigue desde la presión en las boquillas desde 3 bar hasta 0,6 bar, con una gran uniformidad en la distribución del agua en la parcela. Además se disminuyen las pérdidas por evaporación gracias al tipo de gota producida.

La energía solar fotovoltaica para riego se genera en instalaciones para autoconsumo puro, sin conexión a la red de las compañías eléctricas, no estando sujeta a ningún impuesto o gravamen. También puede resultar interesante utilizar un sistema híbrido de fotovoltaica y diésel, o conexión a la red eléctrica en los períodos de tarifa valle.

\section{Descripción del funcionamiento de la instalación}

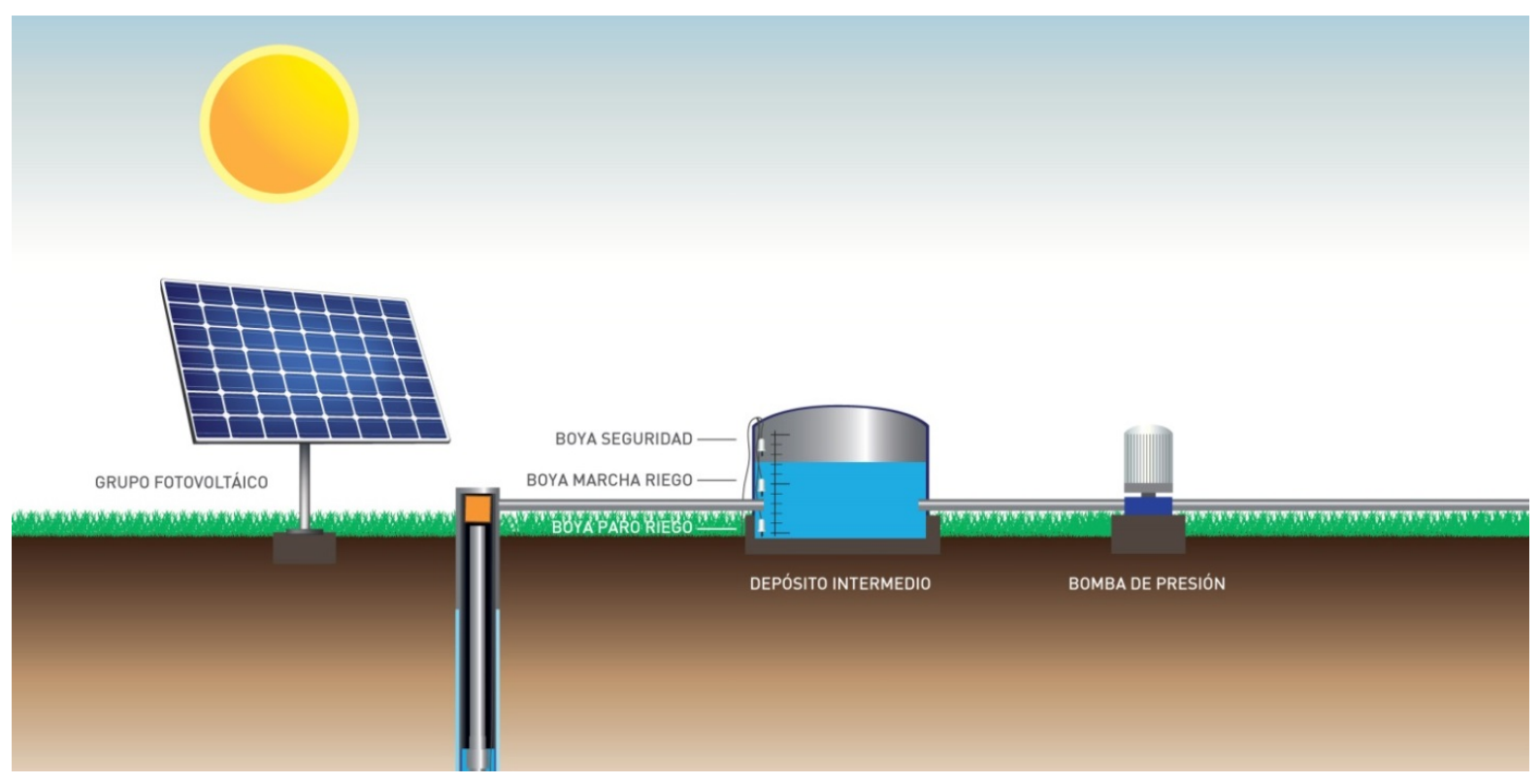

Figura 1: Esquema de la instalación 
Se ha realizado una instalación fotovoltaica, en la que se han empleado $800 \mathrm{~m}^{2}$ de paneles solares, que proporcionan una potencia de $120 \mathrm{~kW}-\mathrm{p}$, capaces de abastecer el bombeo de una explotación representativa de Castilla y León, con una superficie total en riego de 70 ha, correspondientes a cultivos como remolacha azucarera, guisante y alubia verde, patata, maíz, colza y cereales. En total se riegan unas 51 ha de cultivos de primavera y 19 de cultivos de verano, en algunos períodos coinciden en riego las 70 ha.

La energía solar es captada por los paneles solares, que están colocados en la cubierta de una nave, aunque también podrían estar colocados sobre el suelo. Los paneles fotovoltaicos producen corriente continua, que es necesario transformar en corriente alterna, esto se consigue sin utilizar inversores, mediante los propios variadores de frecuencia que alimentan las dos bombas del sistema, sin utilización de baterías, a través de un cuadro de control. Desde este cuadro de control que cuenta con seguimiento del punto de máxima potencia, un autómata o PLC programado será el encargado de, contando con la información que facilitan las boyas de nivel del pozo y del depósito y en función de la radiación disponible en cada momento poner en funcionamiento las bombas por separado o a la vez.

Según se muestra en la figura 2, el agua se bombea mediante dos bombas, una bomba sumergida que funciona a régimen variable y extrae el agua desde un sondeo hasta un pequeño depósito de regulación, y otra bomba exterior que funciona a régimen fijo e impulsa el agua con caudal y presión constante y adecuado a cada sector de riego.

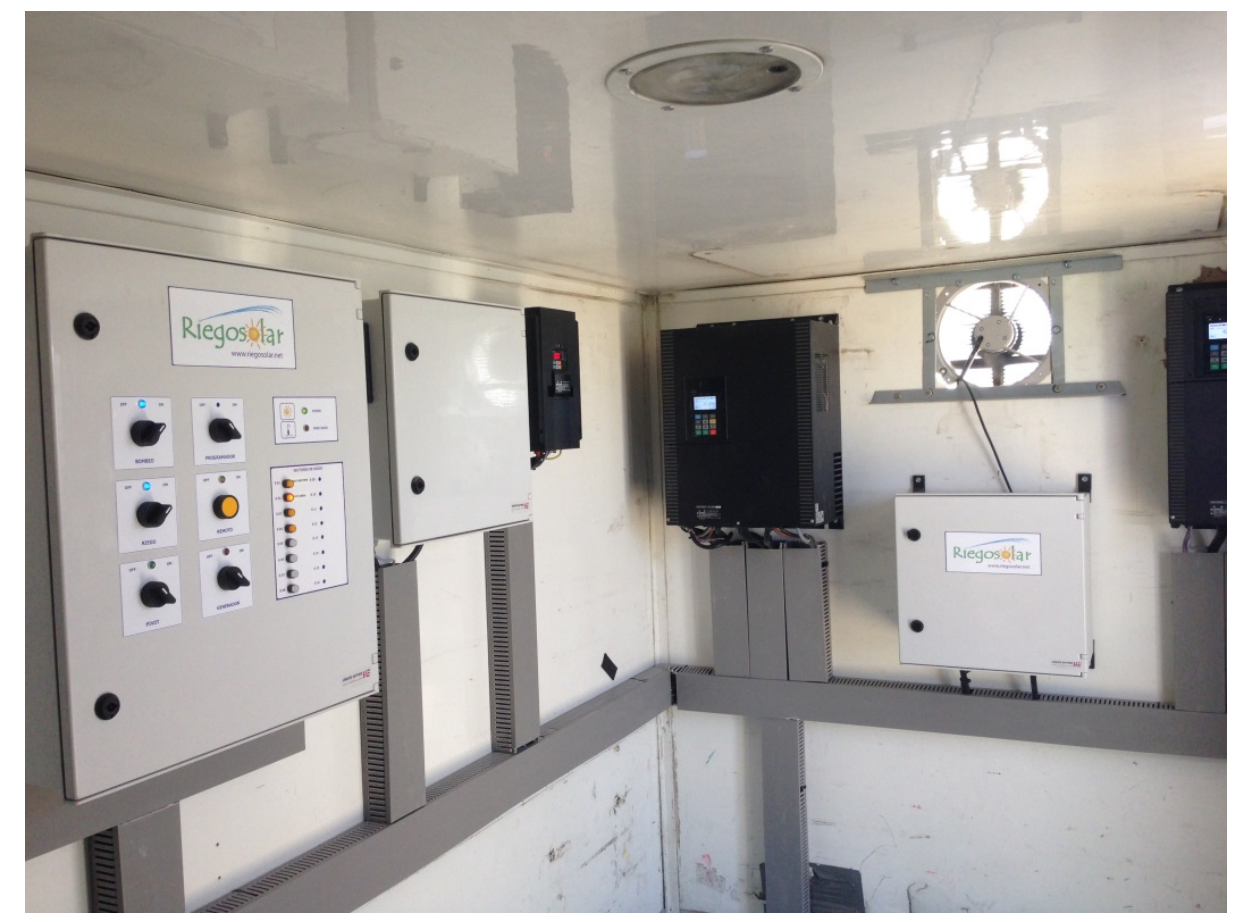

Figura 2: Cuadros de control y variadores de frecuencia

La energía se genera en una instalación fotovoltaica de autoconsumo aislada de la red eléctrica. No se utilizan baterías. Desde el cuadro de control (figura 2), que cuenta con seguimiento del punto de máxima potencia, un autómata o PLC programado es el encargado de optimizar el uso de la energía gestionando el régimen de funcionamiento de cada bomba en cada momento. Los mismos paneles alimentan los motores responsables del movimiento de los pivotes de riego y del resto de aparatos eléctricos, por lo que el sistema es totalmente autónomo. 
El equipo se completa con automatismos y telecontrol que permiten la programación del riego mediante sectores de forma automática y remota desde cualquier dispositivo móvil con internet, utilizando una aplicación (App) propia.

\section{Características de la instalación realizada en Tordesillas}

Superficie regada: 19 ha de cultivos de verano y 51 ha de cultivos de primavera

- Nivel dinámico del agua en el sondeo: $90 \mathrm{~m}$

- Instalaciones de riego: 3 pivotes (56 ha) y 3 coberturas a 12x18 (14 ha)

- Potencia fotovoltaica instalada: $121.600 \mathrm{Wp}$

- Emisiones de $\mathrm{CO}_{2}$ : 0 -cero- frente a 120 t/año de $\mathrm{CO}_{2}$ que se producían cuando se utilizaba gasóleo.

- Capacidad de bombeo: $150.000 \mathrm{l} / \mathrm{hora}$

- Horas de funcionamiento continuo: 10 a 12 horas/día durante la temporada de riego

- Presión del agua en los emisores: en cobertura se riega a 4,5 bar y en pivotes a 1,5 bar a la salida de la bomba.

\section{Rentabilidad}

Sin duda el gran interés que este sistema despierta entre los agricultores españoles es la posibilidad de reducir a menos de la cuarta parte del coste del riego, tanto en el bombeo como la impulsión.

En el grafico 2 se muestra la evolución histórica de precios de gasóleo agrícola desde 1996 hasta la actualidad, vemos como el precio se duplica aproximadamente cada 10 años, por lo que suponemos un incremento anual de un $7 \%$.

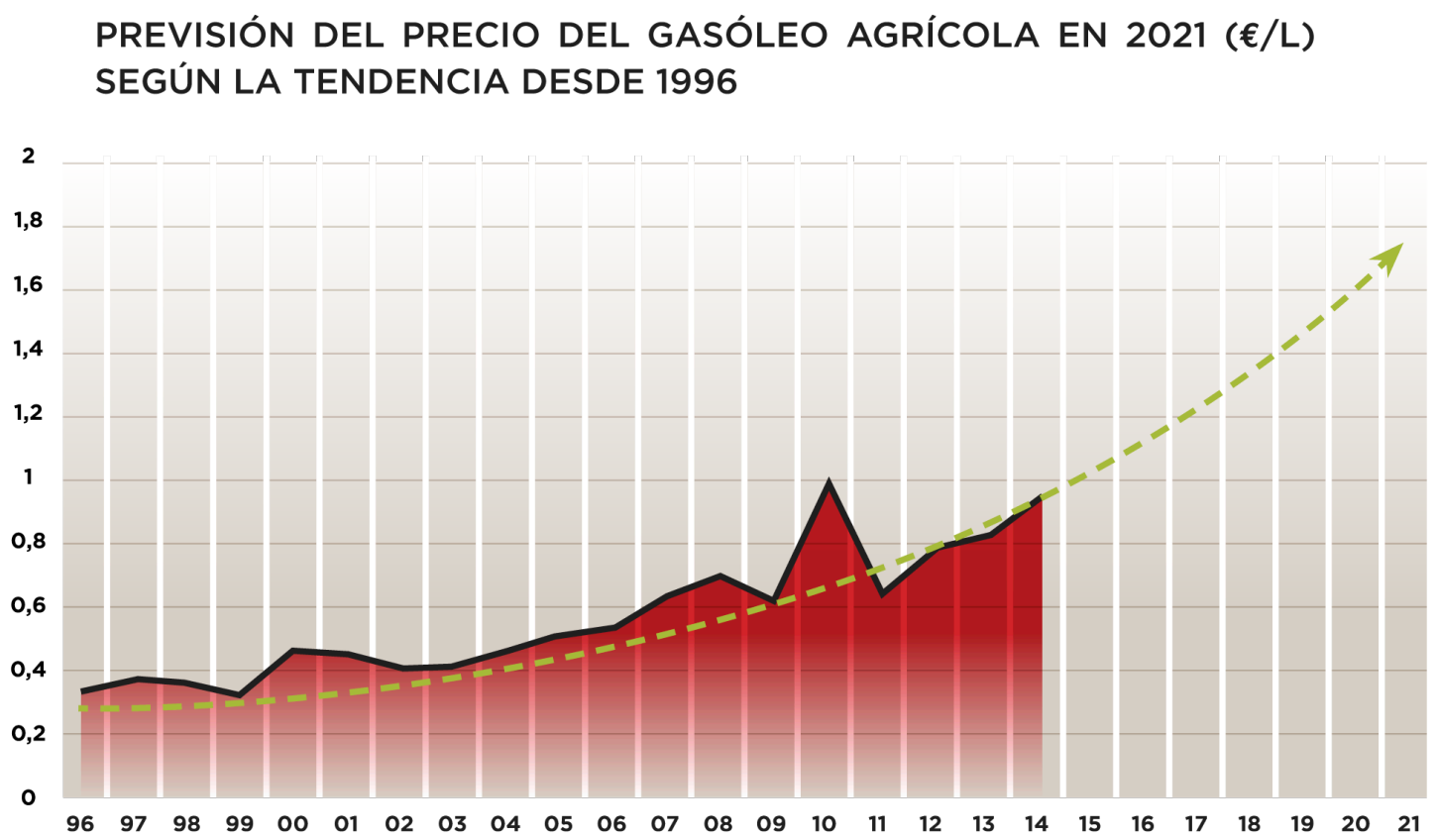

Grafico 2: Histórico y proyección sobre el precio del gasóleo agrícola

Para calcular la rentabilidad, se ha realizado el estudio de costes basado en el demostrador que se ha ejecutado en Tordesillas, comparando los costes del agua antes y después de ejecutar el proyecto. 
En un año normal, el volumen de agua de riego necesario para atender los cultivos de esta explotación es de $240.730 \mathrm{~m}^{3}$ anuales, de estos $111.400 \mathrm{~m}^{3}$ corresponden a 19 ha de riego intensivo (remolacha y guisante-alubia) y $129.300 \mathrm{~m}^{3}$ a 451 ha de riego de cultivos de primavera (trigo, colza y cebada). En la figura 3 se muestra el depósito y las placas solares sobre la nave.

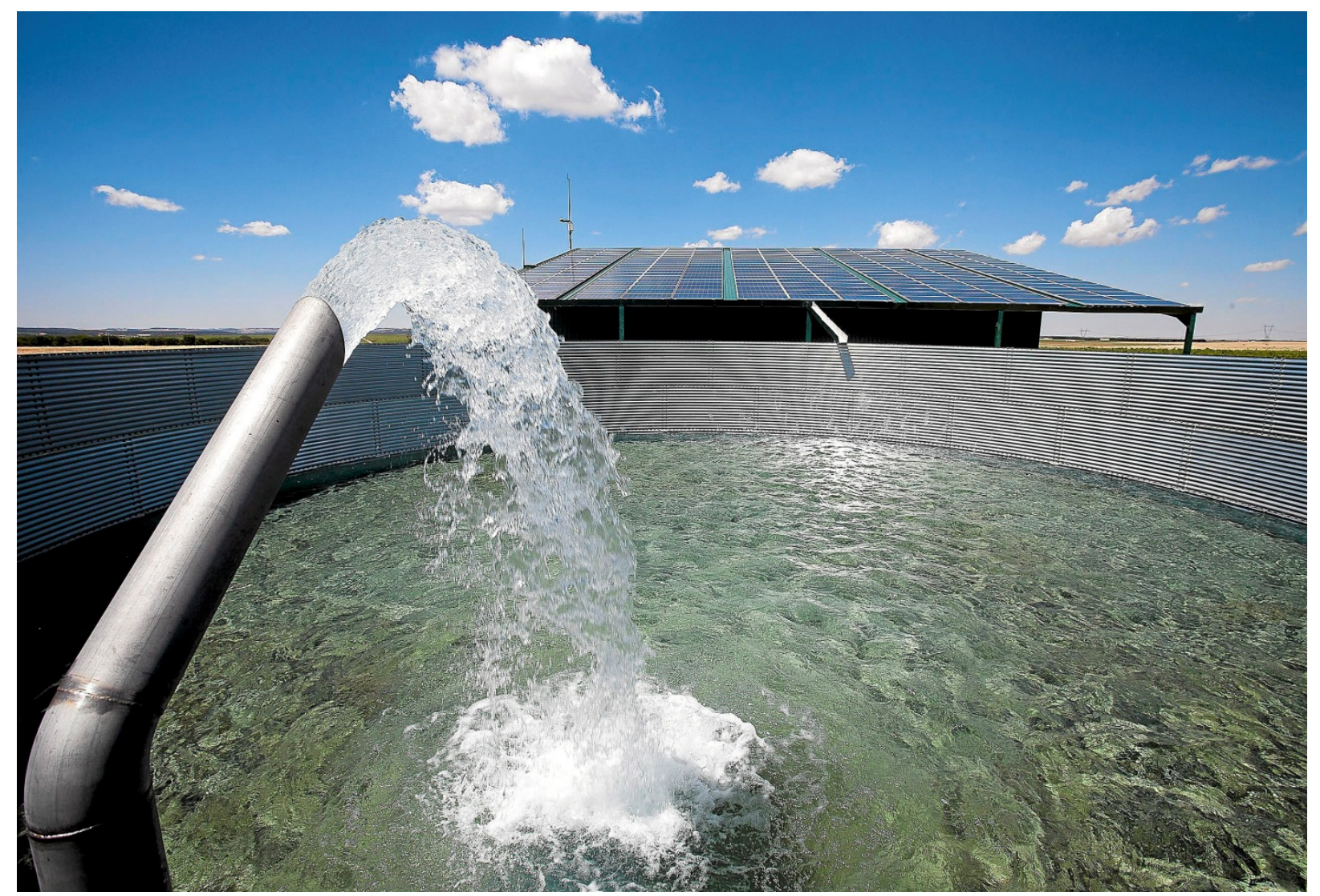

Figura 3: Depósito y nave con las placas solares en la cubierta.

Bombeando con generador diésel el coste de energía en la explotación de Tordesillas ascendió en el año 2013 a $40.664 €$ anuales, lo que equivale a un coste energético de 17 céntimos de euro por $\mathrm{m}^{3}$ de agua de riego, al que habría que sumarle la amortización del generador y de la bomba, estimado en 1,5 a 2 céntimos de $€ / \mathrm{m}^{3}$ ).

Mediante bombeo solar se ha pasado a un coste del agua de 6 céntimos de $€ / \mathrm{m}^{3}$ (si se realiza la amortización de la inversión en un plazo de 12 años. El importe de la inversión asciende a 152.000 euros. En realidad la vida útil de la instalación es muy superior, los fabricantes de paneles solares garantizan un rendimiento de al menos el $80 \%$ al cabo de 20 años; en España es todavía es posible ver las primeras instalaciones fotovoltaicas domésticas, que siguen funcionando después de más de 30 años.

Queda claro que la diferencia de coste entre utilizar la energía convencional o energía solar para el riego es muy grande, pues el plazo de recuperación de la inversión se calcula entre 4 y 5 años, siendo similar la rentabilidad para todo tipo de captaciones de agua, ya se trate de perforaciones o sondeos profundos como de aguas superficiales de pozos, ríos o canales, en las que los requerimientos de potencia son menores, pues en esos casos aunque el ahorro es menor, también es mucho menor la potencia requerida y por tanto la inversión a realizar. 


\section{Valoración económica de la instalación de Tordesillas}

- Coste de la instalación: $152.000 €$

- Coste del agua bombeada: $0,06 € / \mathrm{m}^{3}$ frente a los $0,17 € / \mathrm{m} 3$ cuando se utilizaba el generador de gasóleo. Nota: no existe factura de energía, los 6 céntimos corresponden a la amortización de la instalación en un periodo de 12 años, aunque la vida útil es mucho mayor.

- Plazo de recuperación de la inversión: 4,5 años

\section{Conclusiones}

Con el coste actual de las energías convencionales no renovables y con las subidas que se prevén para el futuro, el riego solar es sin duda una buena solución para conseguir mejorar la rentabilidad de los cultivos de regadío en España y otros países. Pero además del aspecto económico, existen indicadores que nos hacen pensar que el bombeo solar ocupará un papel principal en el riego del futuro, pues permite eliminar por completo las emisiones de $\mathrm{CO}^{2}$ a la atmósfera y proporciona autonomía energética al agricultor. Resultará especialmente útil en aquellos países del tercer mundo o en vías de desarrollo en los que se carece de infraestructuras para el transporte energético.

Dadas las grandes ventajas del bombeo solar, se prevé que durante los próximos años se producirá una rápida difusión de este tipo de tecnología entre los agricultores, de hecho en el primer semestre 2015 se han construido seis nuevas instalaciones con potencias superiores a los $100 \mathrm{~kW}$ en la provincia de Valladolid.

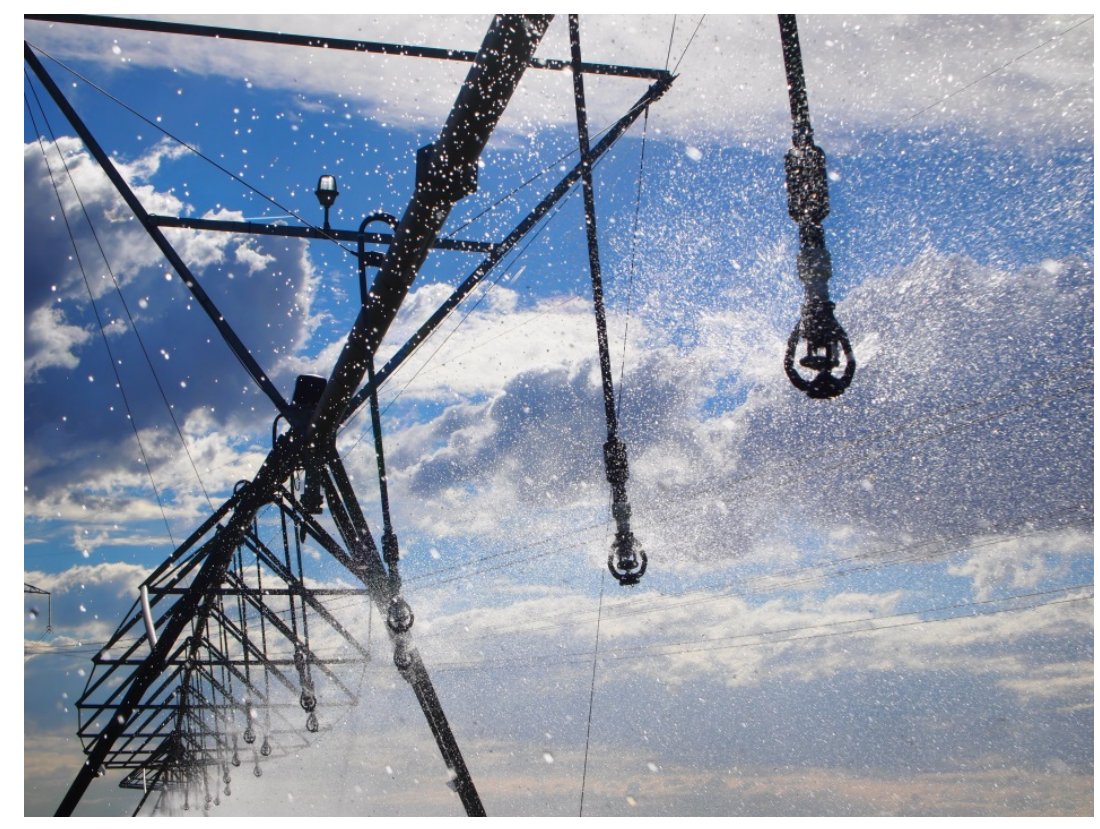

Figura 4: Pivote con emisores I-Wobs de baja presión (6 psi). 


\section{Agradecimientos}

A todas las empresas que han colaborado o apoyado este proyecto: Azucarera, RKD, Senninger, Krannich,solar, Omron, Grundfos, Silver Hidráulica, Regaber, Raesa, Vyrsa e ITC. 